\title{
Models for control of thermal energy in buildings
}

\author{
Casper Amandus Johansen, Bernt Lie, Nils-Olav Skeie \\ Department of Electrical Engineering, Information Technology and Cybernetics \\ University of South-Eastern Norway, Porsgrunn, Norway, Bernt.Lie@usn.no
}

\begin{abstract}
A large fraction of the world's energy production is used for HVAC in buildings. It is therefore important to develop improved strategies for the efficient use of energy in buildings. Storage of intermittent energy production is important; storage as hot water in water tanks is the most common way to store energy in private homes/ smaller apartment complexes. Finding good models for building thermal behavior is an important part of developing building energy management systems (BEMS) that are capable of reducing energy consumption for space heating through model predictive control (MPC). In this paper, previous models of temperature dynamics in hot water tanks are considered, and a simple well mixed tank model is compared with a model describing a more realistic stratified temperature distribution. Two models are fitted to experimental data from a hot water tank. Description of temperature stratification requires a distributed model, but a relatively low order discretized model suffices to describe the important effect while simultaneously being useful for BEMS. A suitable hot water tank model in combination with weather forecast enables temperature estimation and prediction in MPC, and allows for finding a suitable water temperature at minimal energy consumption.
\end{abstract}

Keywords: Energy in buildings, energy storage, hot water tank model, well mixed tank model, stratified flow model, experimental data, model fitting.

\section{Introduction}

\subsection{Background}

A large part of the world's energy production is used for heating/cooling, ventilation, and air conditioning of buildings (HVAC), (Pérez-Lombard et al., 2008), and this fraction is increasing. Even though modern building techniques make it possible to reduce the energy used for heating, the renewal rate of buildings is low. Berthou et al. (Berthou et al., 2014) report renewal rates of $1 \%$ per year in France, with similar rates for other European countries. This illustrates the need for good building energy management systems (BEMS) also in existing buildings.

Model predictive control (MPC) is an attractive approach for use in BEMS. Models of the building thermal behavior can be used to predict the heating and cooling time, and the usage of energy. In an MPC system, a model is used to simulate the system ahead in time in order to find a sequence of inputs that controls the system to the desired state. In a BEMS, the use of MPC will allow for improved control of the indoor climate as well as minimization the energy consumption (Berthou et al., 2014), (Fux et al., 2012). Predictions of future system inputs such as outdoor temperature, irradiation, precipitation, etc., are readily available from internet services, which helps to facilitate the use of MPC.

An important problem in BEMS involves the possibility to store surplus energy for later use. Energy storage as sensible heat in a water tank is a widespread strategy. A simple, yet reasonably accurate water tank model, which can be integrated in a complete building model, is required for successful MPC.

\subsection{Previous work}

In (de Oliveira et al., 2013), a house heating system is optimized wrt. fluctuating energy prices. In (Lie et al., 2014), a related heating system with irradiation prediction, solar collector, and a simple water storage tank is considered. (Lie, 2015) discusses a more detailed model of the water storage tank. (Xu et al., 2014) discuss a more realistic water storage tank, using a simplified description of water buoyancy presented in (Viskanta et al., 1977). (Koch, 2012) discusses both a concentrated and a distributed water tank model, while (Vrettos, 2016) extends on Koch's work with a buoyancy description model. (Johansen, 2019) adjusted the model from (Xu et al., 2014), and fitted the model to experimental data. The results of (Johansen, 2019) are discussed in this paper.

\subsection{Outline of paper}

Section 2 provides a discussion of the system discussed, Section 3 gives an overview of the dynamic model of the water tank, Section 4 gives results from model simulation and fitting of the model to experimental data, with validation. The results are discussed in Section 5, with some conclusions and indications of future work.

\section{Experimental Rig}

\subsection{System Description}

The building under study is a two floor residential building located in the eastern part of Norway, built in 2017. The building was build based on the Norwegian TEK17 regulation and contains a BEMS based on a web based Programmable Logic Controller (PLC). The PLC uses an 
Table 1. Summary of instruments in Figure 1.

\begin{tabular}{cl}
\hline Label & Description \\
\hline P1 & Pump driving water flow in external loop \\
F1 & Volumetric flow rate sensor in external loop \\
V1 & Three way shunt valve for directing water \\
& through water tank \\
V2 & Valve for directing external loop water \\
& through room \\
T1 & Temperature of mixture after valve V1 \\
T2 & Upper temperature sensor in hot water tank \\
T3 & Lower temperature sensor in hot water tank \\
T4 & External loop temperature at entrance to hot \\
& water tank \\
T5 & Ambient temperature, not shown in \\
& Figure 1 \\
H1 & Heating element for hot water tank, 15kW \\
\hline
\end{tabular}

internet based weather service for prediction of outside temperatures and solar radiation parameters. The heating system consists of floor pipes with hot water in each room, and the temperature is adjusted individually by a valve controlling the flow of how water in a specific section. The valves are controlled by the PLC. The floor material in both floors is concrete. A pump, common for all the pipe sections, provides the circulation of hot water in the pipes. An overview of the heating system is shown in Figure 1.

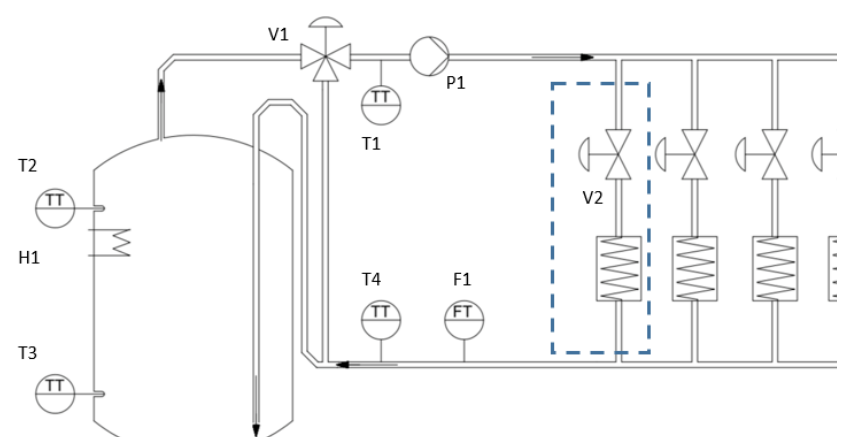

Figure 1. Overview of heating system in building, with hot water buffer tank for heating the water, and floor heating pipes for each room in the building. Each pipe has a valve controlled by the BEMS. The hot water tank is to the left in the figure.

Elements in Figure 1 are summarized in Table 1.

\subsection{Experimental Data}

The PLC system provides measurements of temperatures in the hot water tank (T2, T3), the loop circulation hot mixture temperature (T1), and loop return temperature (T4) once per minute together with control signals for the heating valve (H1) and the three-way valve actuator (V1). Other data such as ambient temperature (T5) and loop flow rate (F1) are sampled more rarely, but have been re-sampled to once per minute. The data have been collected in CSV files with one line for each sample with a time stamp and the measured values. The data set contains data for the period February 5, 2019 to February 21, 2019. Python was used as the software for preprocessing the data, calibration of the models, and validation checks.

\section{Model Description}

\subsection{Model overview}

A model of the buffer tank that can be used in the PLC system is wanted so a model that is adjusted to the computational power of this control system. An overview of the buffer tank is shown in Figure 2.

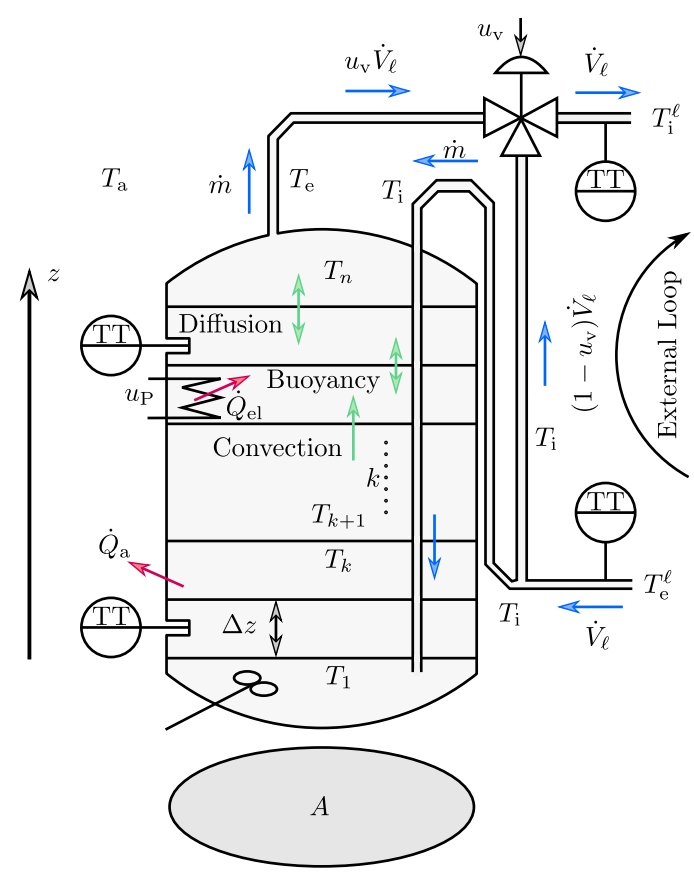

Figure 2. The buffer tank with the heating element, the temperature sensors inside the buffer tank, the temperature sensors on the outlet an inlet pipes and the three way shunt valve for mixing the water from the buffer tank and the return water flow.

Two approaches are used, one model for the tank as a mixed storage tank and a model of the tank as a stratified storage tank where each layer is modeled. Both models are developed based on the macroscopic thermal energy balance, and assuming constant mass/constant mass density. Constant mass $m$ in the system implies that

$$
\dot{m}=\dot{m}_{\mathrm{i}}=\dot{m}_{\mathrm{e}}
$$

where $\dot{m}$ is mass flow rate through the system, while $\dot{m}_{\mathrm{i}}$ and $\dot{m}_{\mathrm{e}}$ are influent and effluent mass flow rates, respectively. The thermal energy balance can be posed as

$$
\frac{\mathrm{d} U}{\mathrm{~d} t}=\dot{H}_{\mathrm{i}}-\dot{H}_{\mathrm{e}}+\dot{W}_{\mathrm{f}}-\dot{W}_{\mathrm{v}}+\dot{Q},
$$

where $U$ is internal energy, $H$ is enthalpy, $W$ is work, and $Q$ is heat. A dot decoration on a symbol indicates a flow rate, thus $\dot{H}$ is enthalpy flow, $\dot{W}$ is mechanical power, and 
$\dot{Q}$ is heat flow rate. $\dot{H}_{\mathrm{i}}$ and $\dot{H}_{\mathrm{e}}$ are influent and effluent enthalpy flow rates, respectively. $\dot{W}_{\mathrm{f}}$ and $\dot{W}_{\mathrm{v}}$ are friction work rate (heating) and power due to volume change $\left(p \frac{\mathrm{d} V}{\mathrm{~d} t}\right)$, respectively; we will neglect friction work, and with constant volume, there is no volume work. Heat flow might be due to added electric heating $\dot{Q}_{\mathrm{el}}$, heat diffusion $\dot{Q}_{\mathrm{d}}$, and heat loss to the ambient $\dot{Q}_{\mathrm{a}}$.

Enthalpy is an extensive quantity, hence for a pure substance,

$$
H=m \hat{H}
$$

where $\hat{H}$ is specific enthalpy. Likewise, enthalpy flow for a pure substance is related to mass flow as

$$
\dot{H}=\dot{m} \hat{H} .
$$

For an in-compressible liquid, $\hat{H}$ can be posed as

$$
\hat{H}=\hat{H}\left(T^{\circ}\right)+\hat{c}_{p, \mathrm{w}}\left(T-T^{\circ}\right)+\frac{1}{\rho}\left(p-p^{\circ}\right)
$$

where $T^{\circ}$ and $p^{\circ}$ are standard state temperature and pressure, respectively, and $\rho$ is the water density. Under normal conditions, we can neglect the pressure effect. Also, for a pure substance (non-reacting) system, the standard state specific enthalpy $\hat{H}\left(T^{\circ}\right)$ can be neglected. ${ }^{1}$ We will also utilize that for water in liquid form, $\mathrm{d} U \approx \mathrm{d} H$.

\subsection{Mixed tank model}

The first model is a simplified model assuming that the entire volume of the tank is well mixed. The assumptions for the mixed tank model are (1) the top and bottom of the tank are assumed to be horizontal, (2) water flows only from the bottom to the top of the tank, (3) constant density of the water, (4) temperature independent heat capacity, (5) a proportional relationship between the inflow and outflow of the tank and the valve openings, (6) the temperature is homogeneous along the height of the tank.

Based on the assumptions indicated in Section 3.1, we have

$$
\begin{aligned}
\frac{\mathrm{d} U}{\mathrm{~d} t} & \approx \frac{\mathrm{d} H}{\mathrm{~d} t}=\frac{\mathrm{d}}{\mathrm{d} t}(m \hat{H})=m \frac{\mathrm{d} \hat{H}}{\mathrm{~d} t}=m \hat{c}_{p, \mathrm{w}} \frac{\mathrm{d} T}{\mathrm{~d} t} \\
\dot{H}_{\mathrm{i}}-\dot{H}_{\mathrm{e}} & =\dot{m} \hat{c}_{p, \mathrm{w}}\left(T_{\mathrm{i}}-T\right)
\end{aligned}
$$

where we have used that for a perfectly mixed tank, $T_{\mathrm{e}}=$ $T$. For a well mixed tank, there is no heat diffusion. Added electric heating is

$$
\dot{Q}_{\mathrm{el}}=P^{\circ} u_{\mathrm{P}}
$$

where $P^{\circ}[\mathrm{kW}]$ is the maximum electric heating power, and $u_{\mathrm{P}} \in[0,1]$ is a control signal. Heat added from the surroundings is

$$
\dot{Q}_{\mathrm{a}}=\mathscr{U} A_{\mathrm{s}}\left(T_{\mathrm{a}}-T\right)
$$

${ }^{1} \hat{H}\left(T^{\circ}\right)$ is mainly needed for finding heat of formation in chemical reactions. where $\mathscr{U}$ is the overall heat transfer coefficient and $A_{\mathrm{s}}$ is the total surface areas, while $T_{\mathrm{a}}$ is the ambient temperature. The mass flow rate $\dot{m}$ through the hot water tank is given by $\dot{m}=\rho \dot{V}$ where the volumetric flow rate, $\dot{V}$, is given by a split-range control signal $u_{\mathrm{v}}$ as $\dot{V}=\dot{V}_{\ell} u_{\mathrm{v}}$, with $\dot{V}_{\ell}$ being the volumetric flow rate in the external loop. The mass flow rate can thus be expressed as

$$
\dot{m}=\rho \dot{V}_{\ell} \cdot u_{\mathrm{v}}
$$

where $\rho$ is water density, while $u_{\mathrm{v}} \in[0,1]$ is the valve signal.

With $m=\rho V$ and $V$ the tank volume, the model can thus be summarized in state space form as

$$
\begin{aligned}
m \hat{c}_{p, \mathrm{w}} \frac{\mathrm{d} T}{\mathrm{~d} t}=\dot{m} \hat{c}_{p, \mathrm{w}}\left(T_{\mathrm{i}}-T\right)+P^{\circ} u_{\mathrm{P}}+\mathscr{U} A_{\mathrm{s}}\left(T_{\mathrm{a}}-T\right) \\
\Downarrow \\
\frac{\mathrm{d} T}{\mathrm{~d} t}=\frac{\dot{V}_{\ell} \cdot u_{\mathrm{v}}}{V}\left(T_{\mathrm{i}}-T\right)+\frac{P^{\circ} u_{\mathrm{P}}+\mathscr{U} A_{\mathrm{s}}\left(T_{\mathrm{a}}-T\right)}{\rho V \hat{c}_{p, \mathrm{w}}} .
\end{aligned}
$$

\subsection{Stratified tank model}

\subsubsection{Distributed parameter model}

We consider a well mixed volume $\Delta V=A \cdot \Delta z$ in the water tank, where $A$ is the cross sectional area and $\Delta z$ is the height of the volume, with $z=0$ at the bottom of the tank and $z=h$ at the top of the tank. For this volume and with $\mathrm{d} U \approx \mathrm{d} H$, influent at position $z$, effluent at position $z+\Delta z$, the energy balance is

$$
\frac{\mathrm{d} H_{\mathrm{z}+\Delta \mathrm{z}}}{\mathrm{d} t} \approx \dot{H}_{z}-\dot{H}_{z+\Delta z}+\dot{Q}_{\mathrm{el}, \Delta z}+\dot{Q}_{\mathrm{d}, z}-\dot{Q}_{\mathrm{d}, z+\Delta z}+\dot{Q}_{\mathrm{a}, \Delta z}
$$

The following expressions are valid except at the boundaries, i.e., they are valid for $z \in(0, h)$ :

$$
\begin{aligned}
H_{z+\Delta z} & =m_{\Delta z} \hat{H}_{z+\Delta z}=\rho A \Delta z \cdot \hat{c}_{p, \mathrm{w}}\left(T_{z+\Delta z}-T^{\circ}\right) \\
\dot{H}_{z}-\dot{H}_{z+\Delta z} & =\dot{m} \hat{c}_{p, \mathrm{w}}\left(T_{z}-T_{z+\Delta z}\right) \\
\dot{Q}_{\mathrm{el}, \Delta z} & =P^{\circ} \cdot 1 \mathscr{P}(z) \cdot u_{\mathrm{P}} \\
\dot{Q}_{\mathrm{d}} & =A \dot{Q}_{\mathrm{d}}^{\prime \prime} \\
\dot{Q}_{\mathrm{a}, \Delta z} & =\mathscr{U} A_{\Delta z}\left(T_{\mathrm{a}}-T_{z+\Delta z}\right) .
\end{aligned}
$$

In these expressions, we have assumed that the heating element is located at a point position $z_{\mathrm{P}}$, and $1_{\mathscr{P}}(z)$ is the indicator function defined as

$$
1_{\mathscr{P}_{\Delta z}}(z)= \begin{cases}1, & z \in \mathscr{P}_{\Delta z} \\ 0, & z \notin \mathscr{P}_{\Delta z},\end{cases}
$$

with heating element location set $\mathscr{P}_{\Delta z}$ given as

$$
\mathscr{P}_{\Delta z}=\left(z_{\mathrm{P}}, z_{\mathrm{P}}+\Delta z\right] \text {. }
$$

Furthermore, $\dot{Q}_{\mathrm{d}}^{\prime \prime}$ is the heat diffusion per unit cross sectional area (the heat flux), while $A_{\Delta z}=\wp \Delta z$ and $\wp$ is the perimeter of the tank. 
By combining these terms into the thermal energy balance, dividing by $\Delta z$ and letting $\Delta z \rightarrow 0$, we find that for $z \in(0, h)$ :

$$
\begin{aligned}
\rho A \hat{c}_{p, \mathrm{w}} \frac{\partial T}{\partial t} & =-\dot{m} \hat{c}_{p, \mathrm{w}} \frac{\partial T}{\partial z}+P^{\circ} \delta\left(z-z_{\mathrm{P}}\right) \cdot u_{\mathrm{P}} \\
& -A \frac{\partial \dot{Q}_{\mathrm{d}}^{\prime \prime}}{\partial z}+\mathscr{U} \wp\left(T_{\mathrm{a}}-T\right),
\end{aligned}
$$

where we have introduced Dirac's delta function $\delta\left(z-z_{\mathrm{P}}\right)$ by observing that

$$
\lim _{\Delta z \rightarrow 0} \frac{1 \mathscr{P}_{\Delta z}(z)}{\Delta z} \rightarrow \delta\left(z-z_{\mathrm{P}}\right) .
$$

The heat flux $\dot{Q}_{\mathrm{d}}^{\prime \prime}$ consists of two terms:

1. Thermal diffusion flux $\dot{Q}_{\mathrm{d}, \mathrm{d}}^{\prime \prime}$ given by Fourier's law,

$$
\dot{Q}_{\mathrm{d}, \mathrm{d}}^{\prime \prime}=-k_{\mathrm{t}} \frac{\partial T}{\partial z}
$$

where $k_{\mathrm{t}}$ is thermal conductivity and is assumed constant here, and

2. Buoyant turbulent mixing flux $\dot{Q}_{\mathrm{d}, \mathrm{b}}^{\prime \prime}$ given as $(\mathrm{Xu}$ et al., 2014)

$$
\frac{\partial \dot{Q}_{\mathrm{d}, \mathrm{b}}^{\prime \prime}}{\partial z}=-k_{\mathrm{b}} \frac{\partial^{2} T}{\partial z^{2}}
$$

where $k_{\mathrm{b}}$ is buoyant conductivity given as

$$
k_{\mathrm{b}}= \begin{cases}c_{\mathrm{b}} \kappa^{2} d^{2} \sqrt{g \alpha_{p} \mid \frac{\partial T}{\partial z \mid},}, & \frac{\partial T}{\partial z}<0 \\ 0, & \frac{\partial T}{\partial z} \geq 0\end{cases}
$$

where $\kappa$ is the von Karman constant $(\kappa \approx 0.4), d$ is some characteristic length - the diameter in this case, $g$ is the acceleration of gravity, $\alpha_{p}$ is the thermal expansion coefficient at constant pressure, and $c_{\mathrm{b}} \sim 1$ is a tuning factor. Because hot water has lower density than cold water, the normal steady situation is that $T$ is higher at larger $z$ (with the given direction of $z$ ), hence with $\frac{\partial T}{\partial z}>0$ this is the normal situation and there is no buoyancy. On the other hand, with $\frac{\partial T}{\partial z}<0$, the temperature profile is reversed, and buoyancy kicks in. (Vrettos, 2016) gives an alternative expression for buoyancy mixing.

In summary, for $z \in(0, h)$, the model can be simplified to

$$
\begin{aligned}
\frac{\partial T}{\partial t} & =-\frac{\dot{V}_{\ell} \cdot u_{\mathrm{v}}}{A} \frac{\partial T}{\partial z}+\frac{k_{\mathrm{t}}+k_{\mathrm{b}}}{\rho \hat{c}_{p, \mathrm{w}}} \frac{\partial^{2} T}{\partial z^{2}} \\
& +\frac{P^{\circ} \delta(z-z \mathrm{P}) \cdot u_{\mathrm{P}}+\mathscr{U} \wp\left(T_{\mathrm{a}}-T\right)}{\rho A \hat{c}_{p, \mathrm{w}}} .
\end{aligned}
$$

Because of the second derivative in the $z$-direction, we need two boundary conditions. These are

$$
\begin{aligned}
& T_{z=0^{-}}=T_{\mathrm{i}} \\
& T_{z=h^{+}}=T_{z=h} .
\end{aligned}
$$

There is also an additional ambient heat loss surface at the bottom and the top.

The water tank is encased by an insulator of unknown thermal conductivity $k_{\mathrm{i}}$ and unknown thickness $d_{\mathrm{i}}$. Thermal conductivity of insulator typically has a value in the range $k_{\mathrm{i}} \in[10,50] \mathrm{mW} / \mathrm{mK}$ with air at $k_{\text {air }}=25 \mathrm{~mW} / \mathrm{mK}$. The overall heat transfer coefficient $\mathscr{U}$ typically is described by

$$
\mathscr{U}=\frac{1}{\frac{1}{h_{\mathrm{w}}}+\frac{k_{\mathrm{i}}}{d_{\mathrm{i}}}+\frac{1}{h_{\mathrm{a}}}}
$$

where $h_{\mathrm{w}}$ is the heat transfer coefficient between water and the metal surface, while $h_{\mathrm{a}}$ is the heat transfer coefficient between ambient air and the metal surface. The ambient side will exhibit free convection with typical values for $h_{\mathrm{a}}$ being $h_{\mathrm{a}} \in[2.8,23] \mathrm{W} / \mathrm{m}^{2} \mathrm{~K}$. The water side, will however vary between free convection with stagnant water when $u_{\mathrm{v}} \equiv 0$ and typical values of $h_{\mathrm{w}} \in$ $[50,3000] \mathrm{W} / \mathrm{m}^{2} \mathrm{~K}$, and forced convection when $u_{\mathrm{v}} \neq 0$ with $h_{\mathrm{w}} \in[280,17000] \mathrm{W} / \mathrm{m}^{2} \mathrm{~K}$. Typically, if the insulation is $5 \mathrm{~cm}$ thick, $\frac{k_{\mathrm{i}}}{d_{\mathrm{i}}} \in[0.2,1] \mathrm{W} / \mathrm{m}^{2} \mathrm{~K}$.

\subsubsection{Semi-discretized model}

Because the flow of water is specified, it suffices with a simple finite difference discretization of the spatial derivatives. If the water tank height $h$ is divided into $n$ equal height slices, $\Delta z=\frac{h}{n}$ with the bottom slice numbered $k=1$ and the upper slice numbered $k=n$, we have for $k \in\{2, \ldots, n-1\}:$

$$
\begin{aligned}
\frac{\mathrm{d} T_{k}}{\mathrm{~d} t} & =-\frac{\dot{V}_{\ell} \cdot u_{\mathrm{v}}}{A} \frac{T_{k}-T_{k-1}}{\Delta z}+\frac{k_{\mathrm{t}}+k_{\mathrm{b}}}{\rho \hat{c}_{p, \mathrm{w}}} \frac{T_{k+1}-2 T_{k}+T_{k-1}}{\Delta z^{2}} \\
& +\frac{P^{\circ} \frac{1_{\mathscr{P}_{\Delta z}}(k \cdot \Delta z)}{\Delta z} \cdot u_{\mathrm{P}}+\mathscr{U} \wp\left(T_{\mathrm{a}}-T_{k}\right)}{\rho A \hat{c}_{p, \mathrm{w}}} .
\end{aligned}
$$

Here,

$$
\frac{k_{\mathrm{b}}}{\rho \hat{c}_{p, \mathrm{w}}}= \begin{cases}c_{\mathrm{b}} \kappa^{2} d^{2} \sqrt{g \alpha_{p} \mid \frac{T_{k+1}-T_{k} \mid}{\Delta z},} & T_{k}>T_{k+1} \\ 0, & T_{k} \leq T_{k+1}\end{cases}
$$

where $c_{\mathrm{b}} \sim 1$ is a tuning factor.

At the boundaries, the scheme of 29 is invalid, and is modified to:

$k=1:$ For the advection term, $T_{0}$ becomes $T_{\mathrm{i}}$, while for the diffusion term, $T_{0}$ equals $T_{1}$,

$$
\begin{aligned}
\frac{\mathrm{d} T_{1}}{\mathrm{~d} t}= & -\frac{\dot{V}_{\ell} \cdot u_{\mathrm{v}}}{A} \frac{T_{1}-T_{\mathrm{i}}}{\Delta z}+\frac{k_{\mathrm{t}}+k_{\mathrm{b}}}{\rho \hat{c}_{p, \mathrm{w}}} \frac{T_{2}-T_{1}}{\Delta z^{2}} \\
+ & \frac{P^{\circ} \frac{1_{\mathscr{P}_{\Delta z}}(\Delta z)}{\Delta z} \cdot u_{\mathrm{P}}+\mathscr{U}\left(\wp+\frac{A}{\Delta z}\right)\left(T_{\mathrm{a}}-T_{1}\right)}{\rho A \hat{c}_{p, \mathrm{w}}} .
\end{aligned}
$$

$k=n$ : We assume that the temperature of the metal above cell $n$ has the same temperature as cell $n$ because of 


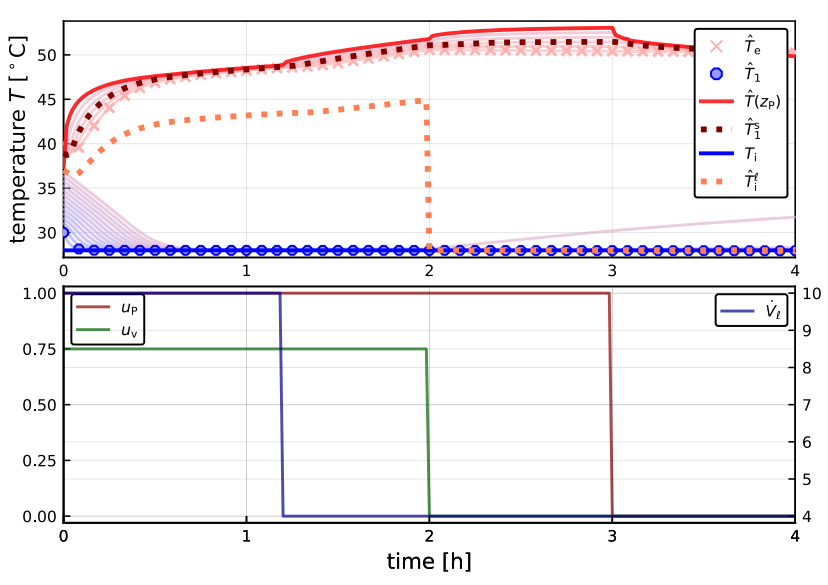

Figure 3. Stratified model simulation. Model parameters are taken from Table 2. Dotted lines indicate simulated temperatures at temperature sensor locations; temperature at inlet layer $\left(T_{1}\right)$ is indicated with $\circ$ markers, and temperature at exit layer $\left(T_{\mathrm{e}}\right)$ is indicated with $\times$ markers.

good thermal conduction in the metal and good insulation, thus $T_{n+1}=T_{n}$. We then have

$$
\begin{aligned}
\frac{\mathrm{d} T_{n}}{\mathrm{~d} t}= & -\frac{\dot{V}_{\ell} \cdot u_{\mathrm{v}}}{A} \frac{T_{n}-T_{n-1}}{\Delta z}+\frac{k_{\mathrm{t}}+k_{\mathrm{b}}}{\rho \hat{c}_{p, \mathrm{w}}} \frac{T_{n-1}-T_{n}}{\Delta z^{2}} \\
& +\frac{P^{\circ} \frac{1_{\mathscr{P}_{\Delta z}}(n \cdot \Delta z)}{\Delta z} \cdot u_{\mathrm{P}}+\mathscr{U}\left(\wp+\frac{A}{\Delta z}\right)\left(T_{\mathrm{a}}-T_{n}\right)}{\rho A \hat{c}_{p, \mathrm{w}}} .
\end{aligned}
$$

\subsection{Model parameters}

Table 2 lists nominal model parameters for the water tank.

In Table 2, it should be observed that $h_{\mathrm{a}}$ and $\frac{k_{\mathrm{i}}}{d_{\mathrm{i}}}$ dominates total over $h_{\mathrm{w}}$, so that $\mathscr{U} \approx 1 /\left(1 / h_{\mathrm{a}}+d_{\mathrm{i}} / k_{\mathrm{i}}\right)$ with both free and forced water convective heat transfer, and $\mathscr{U} \approx 0.43 \mathrm{~W} / \mathrm{m}^{2} \mathrm{~K}$.

\subsection{Operating conditions}

Typical operating conditions for the water tank are given in Table 3.

\subsection{Basic simulation of stratified tank model}

Figure 3 shows the temperature response at $n=20$ positions of the stratified model; $T_{1: n}(0)=[30: 40]^{\circ} \mathrm{C}, T_{\mathrm{a}}=$ $25^{\circ} \mathrm{C}, T_{\mathrm{i}}=28^{\circ} \mathrm{C}, \dot{V}_{\ell}=10 \cdot \mathbb{H}_{0}-6 \cdot \mathbb{H}_{1.2 \mathrm{~h}} \mathrm{~L} / \mathrm{min}, u_{\mathrm{v}}=$ $0.75 \cdot \mathbb{H}_{0}-0.75 \cdot \mathbb{H}_{2 \mathrm{~h}}$ and $u_{\mathrm{P}}=\mathbb{H}_{0}-\mathbb{H}_{3 \mathrm{~h}}$ where $\mathbb{H}_{t}$ is the Heaviside function.

Observe that with default values, the buoyant conductivity is very large if the initial profile of $T_{k}(0)$ is reversed; in Figure 3, $c_{\mathrm{b}}=10^{-2}$ has been used. With the positive initial temperature gradient in Figure 3, there is no buoyancy. With the geometry of the tank and $u_{\mathrm{v}}=0.75$, a "plug" of water entering the tank takes $40 \mathrm{~min}$ to pass through the tank with $\dot{V}_{\ell}=10 \mathrm{~L} / \mathrm{min}$, and $100 \mathrm{~min}$ with $\dot{V}_{\ell}=4 \mathrm{~L} / \mathrm{min}$.

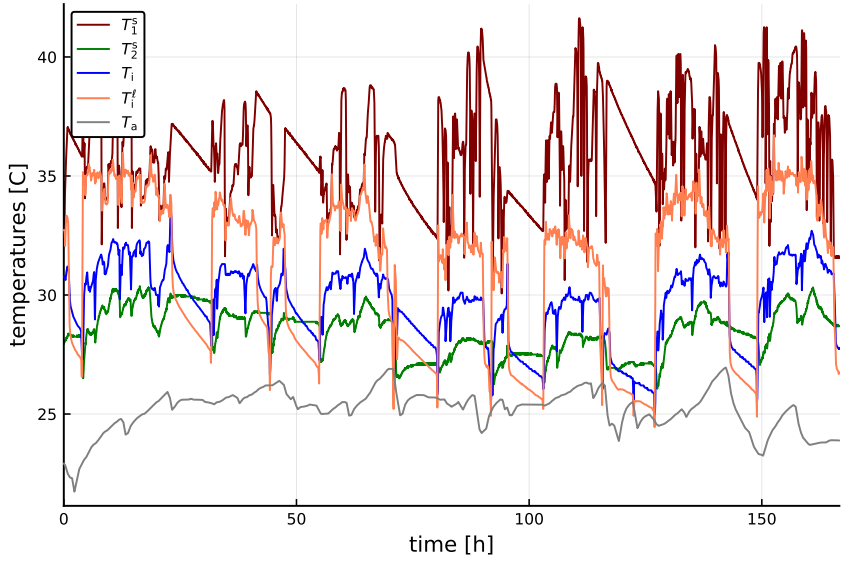

Figure 4. Experimental values of temperatures.

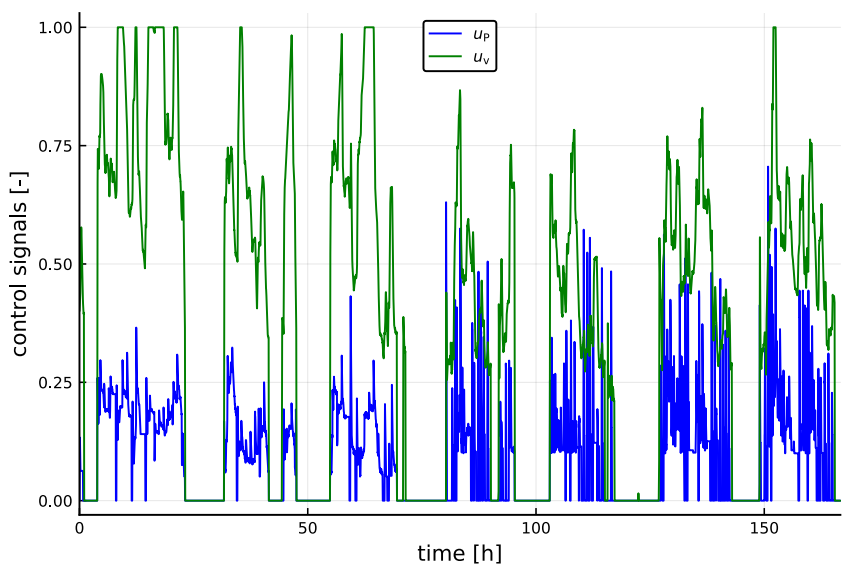

Figure 5. Experimental values for control signals.

\section{Model fitting}

\subsection{Sensor signals and experimental data}

The available sensors for the water tank are listed in Table 4 .

Here, it should be observed that the lower temperature sensor $T_{2}^{\mathrm{s}}$ gives rather uncertain results due to poor insulation from the external metal of the water tank.

The heated loop temperature $T_{\mathrm{i}}^{\ell}$ (T1) in Table 4 is the temperature of the mixture of the effluent water from the water tank and the by-passed water. Thus, using steady energy balance for the 3-way mixing valve, we have

$$
T_{\mathrm{i}}^{\ell}=\left(1-u_{\mathrm{v}}\right) T_{\mathrm{i}}+u_{\mathrm{v}} T_{\mathrm{e}}
$$

where $T_{\mathrm{e}}$ is the effluent temperature from the tank, i.e., $T_{\mathrm{e}}=T$ for the well mixed tank model, and $T_{\mathrm{e}}=T_{n}$ for the stratified tank model.

Figures 4-6 display typical values for the sensor signals, with resolution in $1 \mathrm{~min}$. It should be observed that with $u_{\mathrm{v}} \equiv 0$, according to $33, T_{\mathrm{i}}^{\ell}$ should equal $T_{\mathrm{i}}$. Instead, Figure 4 indicates a bias of ca. $1.1{ }^{\circ} \mathrm{C}$ under that condition.

\subsection{Measure of model fit}

From a system theoretic point of view, $T_{\mathrm{a}}, T_{\mathrm{i}}, u_{\mathrm{P}}$, and $u_{\mathrm{v}}$ are inputs to the dynamic model, while $T_{1}^{\mathrm{s}}, T_{2}^{\mathrm{s}}$, and $T_{\mathrm{i}}^{\ell}$ 
Table 2. Nominal parameters for water tank.

\begin{tabular}{ccl}
\hline Parameter & Value & Comment \\
\hline$g$ & $9.81 \mathrm{~m} / \mathrm{s}^{2}$ & Acceleration of gravity \\
$\kappa$ & $0.41-$ & von Karman constant \\
$\rho$ & $10^{3} \mathrm{~kg} / \mathrm{m}^{3}$ & Water density \\
$\hat{c}_{p, \mathrm{w}}$ & $4.19 \mathrm{~kJ} / \mathrm{kg} \mathrm{K}$ & Specific heat capacity, water \\
$\alpha_{p}$ & $303 \cdot 10^{-6} 1 / \mathrm{K}$ & Thermal expansion coefficient \\
$k_{\mathrm{t}}$ & $0.6 \mathrm{~W} / \mathrm{m} \mathrm{K}$ & Thermal conductivity, water \\
$h$ & $1.5 \mathrm{~m}$ & Height of water column \\
$d$ & $0.5 \mathrm{~m}$ & Internal diameter, tank \\
$\wp$ & $\pi d$ & Perimeter of water tank \\
$A$ & $\pi \frac{d^{2}}{4}$ & Cross sectional area, tank \\
$A_{\mathrm{s}}$ & $2 A+\wp \cdot h$ & Surface area, tank \\
$V$ & $A h$ & Water volume \\
$h_{\mathrm{a}}$ & $3 \mathrm{~W} / \mathrm{m}^{2} \mathrm{~K}$ & Heat transfer, air \\
$h_{\mathrm{w}, \text { free }}$ & $50 \mathrm{~W} / \mathrm{m}^{2} \mathrm{~K}$ & Heat transfer water, free convection \\
$h_{\mathrm{w}, \text { forced }}$ & $1000 \mathrm{~W} / \mathrm{m}^{2} \mathrm{~K}$ & Heat transfer water, forced convection \\
$\frac{k_{\mathrm{i}}}{d_{\mathrm{i}}}$ & $0.5 \mathrm{~W} / \mathrm{m}^{2} \mathrm{~K}$ & Typical value for a 5 cm thick insulator \\
$\mathscr{U}_{\text {free }}$ & $1 /\left(\frac{1}{h_{\mathrm{a}}}+\frac{k_{\mathrm{i}}}{d_{\mathrm{i}}}+\frac{1}{h_{\mathrm{w}, \text { free }}}\right)$ & Overall heat transfer coefficient, free \\
$\mathscr{U}_{\text {forced }}$ & $1 /\left(\frac{1}{h_{\mathrm{a}}}+\frac{k_{\mathrm{i}}}{d_{\mathrm{i}}}+\frac{1}{h_{\mathrm{w}, \text { forced }}}\right)$ & Overall heat transfer coefficient, forced \\
$P^{\circ}$ & $15 \mathrm{~kW}$ & Maximum power of heating element \\
$z_{P}$ & $1.15 \mathrm{~m}$ & Position of heating element \\
\hline
\end{tabular}

Table 3. Typical operating conditions for water tank

\begin{tabular}{ccl}
\hline Variable & \multicolumn{1}{c}{ Value } & Comment \\
\hline$T$ & {$[25,45]^{\circ} \mathrm{C}$} & Water tank temperature \\
$T_{\mathrm{i}}$ & {$[27,33]^{\circ} \mathrm{C}$} & $\begin{array}{l}\text { Tank influent } \\
\text { temperature }\end{array}$ \\
$T_{\mathrm{a}}$ & {$\left[4,27{ }^{\circ} \mathrm{C}\right.$} & $\begin{array}{l}\text { Ambient temperature } \\
\dot{V}_{\ell}\end{array}$ \\
$u_{\mathrm{P}}$ & {$[1,13] \mathrm{L} / \mathrm{min}$} & $\begin{array}{l}\text { Volumetric flow rate in } \\
\text { loop }\end{array}$ \\
& {$[0,1]-$} & $\begin{array}{l}\text { Electric power control } \\
\text { signal }\end{array}$ \\
$u_{\mathrm{v}}$ & {$[0,1]-$} & $\begin{array}{l}\text { Water valve control } \\
\text { signal }\end{array}$ \\
\hline
\end{tabular}

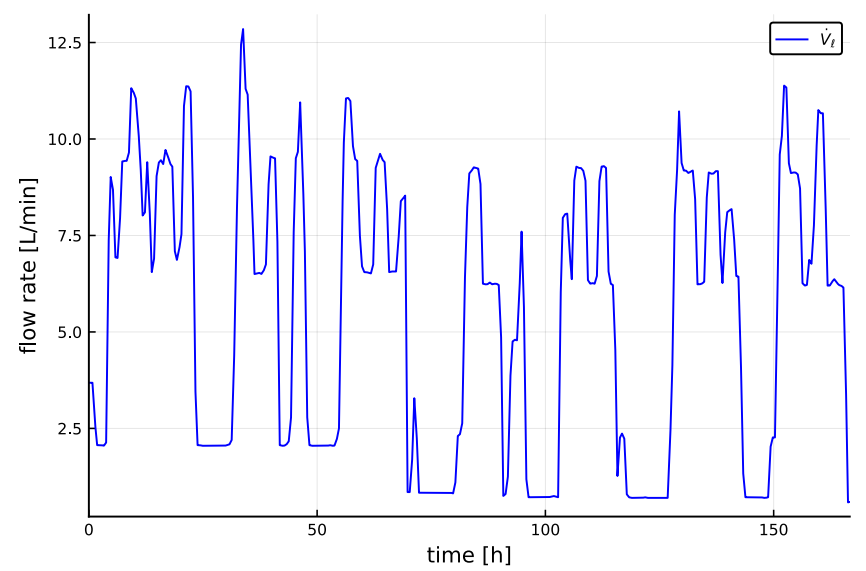

represent measured responses (outputs). Here, $T_{2}^{\mathrm{S}}$ is the lower temperature sensor in the tank, which is reported to be unreliable. Conceptually, we will still include it in the description. To this end, let

$$
u=\left(\begin{array}{cccc}
T_{\mathrm{i}} & T_{\mathrm{a}} & u_{\mathrm{P}} & u_{\mathrm{v}}
\end{array}\right)
$$

be the vector of known inputs to the system, while

$$
y=\left(\begin{array}{ccc}
T_{\mathrm{i}}^{\ell} & T_{1}^{\mathrm{s}} & T_{2}^{\mathrm{s}}
\end{array}\right)
$$

is the vector of output (response) observations. Assume that we have a state space model

$$
\begin{aligned}
\frac{\mathrm{d} x}{\mathrm{~d} t} & =f(x, u ; \theta) \\
y & =g(x, u ; \theta) .
\end{aligned}
$$

In principle we can solve this model such that

$$
y_{t}=G\left(u_{\tau}, x_{0}, \theta\right)
$$

where $y_{t}$ is the model output at time $t$, while $u_{\tau}$ is the input sequence in the interval $[0, t]$. Normally observations are available at discrete time instances $t$; in that case $y_{t}$ is found in discrete time instance $t$ by using a numeric ODE solver.

In general, measured signals have superscript m, i.e., $u^{\mathrm{m}}$ and $y^{\mathrm{m}}$. Introducing the extended parameter set $\tilde{\theta}=\left(\begin{array}{ll}\theta & x(0)\end{array}\right)$, we can measure the model fit by cost function $V(\tilde{\theta})$ given as

$$
V(\tilde{\theta})=\sum_{t=1}^{N}\left\|y_{t}-y_{t}^{\mathrm{m}}\right\|_{W_{y}}^{2}+\lambda \cdot\left\|\tilde{\theta}-\tilde{\theta}^{\circ}\right\|_{W_{\theta}}^{2}
$$


Table 4. Available sensor signals for water tank. Sensor labels (T1-T4, F1) refer to Figure 1.

\begin{tabular}{ccl}
\hline Variable & Unit & Comment \\
\hline$T_{\mathrm{i}}$ & ${ }^{\circ} \mathrm{C}$ & Influent water temperature to tank (T4) \\
$T_{\mathrm{i}}^{\ell}$ & ${ }^{\circ} \mathrm{C}$ & Heated temperature influent to loop (T1) \\
$T_{\mathrm{a}}$ & ${ }^{\circ} \mathrm{C}$ & Ambient temperature \\
$T_{1}^{\mathrm{s}}$ & ${ }^{\circ} \mathrm{C}$ & Water tank temperature at $z_{1}^{\mathrm{s}}=1.3 \mathrm{~m}(\mathrm{~T} 2)$ \\
$T_{2}^{\mathrm{s}}$ & ${ }^{\circ} \mathrm{C}$ & Water tank temperature at $z_{2}^{\mathrm{s}}=0.23 \mathrm{~m}(\mathrm{~T} 3)$ \\
$V_{\ell}$ & $\mathrm{L} / \mathrm{min}$ & Volumetric flow rate in loop $(\mathrm{F} 1)$ \\
$u_{\mathrm{P}}$ & $\frac{\mathrm{V}}{10 \mathrm{~V}}$ & Electric power control signal \\
$u_{\mathrm{v}}$ & $\frac{\mathrm{V}}{10 \mathrm{~V}}$ & Water valve control signal \\
\hline
\end{tabular}

where $\|\cdot\|_{W}$ denotes the weighted 2-norm. Here, $V_{\lambda=0}$ is the standard least squares cost function, while $\lambda>0$ regularizes the problem by emphasizing a prior parameter "guess" $\tilde{\theta}^{\circ}$ which can be based on physical considerations. It is also possible to add hard constraints in the form $\tilde{\theta} \subseteq \tilde{\Theta}$. Typically $W_{y}$ and $W_{\theta}$ are chosen such that the individual elements of vectors have comparable values, e.g., normalized to $[0,1]$ or $[-1,1]$, or standardized to have unit standard deviation. ${ }^{2}$

To assess how well the model with parameters $\hat{\tilde{\theta}}$ generalizes from training data to validation, it is common to compare the root mean squared error (RMSE) $\varepsilon_{\mathrm{RMS}}$ for the parameter estimate $\hat{\tilde{\theta}}$ applied to the training data, compared to the RMSE for the parameter estimate applied to independent validation data;

$$
\varepsilon_{\mathrm{RMS}}=\sqrt{\frac{1}{N} V(\hat{\tilde{\theta}})}
$$

In $39, \lambda$ is a user selected hyper parameter, usually chosen such that the model generalizes well.

\subsection{Model fitting results}

The parameters $\mathscr{U}, k_{\mathrm{t}}$, and $c_{\mathrm{b}}$ are used as fitting parameters together with the unknown initial conditions of unmeasured temperatures. Tuning $k_{\mathrm{t}}$ is related to adding a "heuristic circular mass flow term" in (Koch, 2012). It should be added that numeric discretization in space introduces artificial mixing, with the extreme case of a concentrated model having complete mixing. The parameter $\theta_{\mathrm{c}}$ for the concentrated (well mixed) tank model is

$$
\theta_{\mathrm{c}}=\mathscr{U}
$$

while for the distributed (stratified) model, the parameter is $\theta_{\mathrm{d}}$ given as

$$
\tilde{\theta}_{\mathrm{d}}=\left[\mathscr{U}, k_{\mathrm{t}}, c_{\mathrm{b}}, T_{1}(t=0), \ldots, T_{n}(t=0)\right] .
$$

The cost function is $V$ given by 39 , with $\lambda \equiv 0$.

For the concentrated model, parameter $\mathscr{U}$ is estimated to $\mathscr{U} \in[2.9,62]$, depending on the initial temperature distribution in the tank. This variation in $\mathscr{U}$ depending on

\footnotetext{
${ }^{2}$ If the measurements are pre-scaled, then $W=I$.
}

Table 5. Bounds $\tilde{\Theta}$ and initial guess $\tilde{\theta}^{(0)}$ for parameters during calibration of distributed model.

\begin{tabular}{ccccc} 
& $\mathscr{U}$ & $k_{\mathrm{t}}$ & $c_{\mathrm{b}}$ & $T_{k}(t=0)$ \\
\cline { 2 - 5 }$\tilde{\Theta}$ & {$[0.1,4]$} & {$[0.1,4]$} & {$[0.1,2]$} & {$[17,47]$} \\
$\tilde{\boldsymbol{\theta}}^{(0)}$ & 1 & 0.6 & 1 & $17-47$
\end{tabular}

Table 6. Estimated parameters for distributed (stratified) model. Calibrated and validated RMSE data are taken for $T_{1}^{\mathrm{s}}$.

\begin{tabular}{cccccc}
\hline$n$ & $\mathscr{U}$ & $k_{\mathrm{t}}$ & $c_{\mathrm{b}}$ & RMSE $_{\text {cal }}$ & RMSE $_{\text {val }}$ \\
\hline 3 & 3.72 & 0.1 & 2.0 & 1.47 & 1.5 \\
10 & 4.0 & 0.1 & 2.0 & 1.51 & 1.53 \\
20 & 2.63 & 3.99 & 0.1 & 1.74 & 1.8 \\
50 & 0.94 & 4.0 & 0.1 & 3.22 & 2.76 \\
\hline
\end{tabular}

the initial state of the system indicates that the well mixed model is not very good.

For the distributed model, the parameters have been bounded as in Table 5 .

The estimated parameters for the distributed model are given in Table 6.

Figure 7 shows how the calibrated model fits the experimental data.

Validated model fit is shown in Figure 8.

\section{Discussion and Conclusions}

Suitable models for hot water tanks are important for successful advanced management and control of energy us-

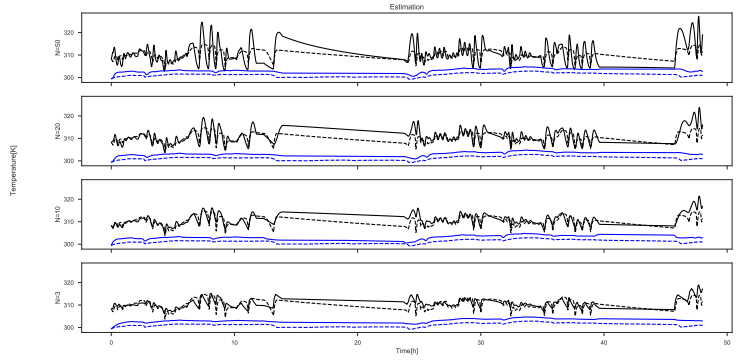

Figure 7. Calibrated model fit for the distributed model. Sensor signals $\left(T_{1}^{\mathrm{s}}, T_{2}^{\mathrm{s}}\right)$ : dashed lines. Simulated result: solid lines. Black color: $T_{1}^{\mathrm{s}}$, blue color: $T_{2}^{\mathrm{s}}$. 


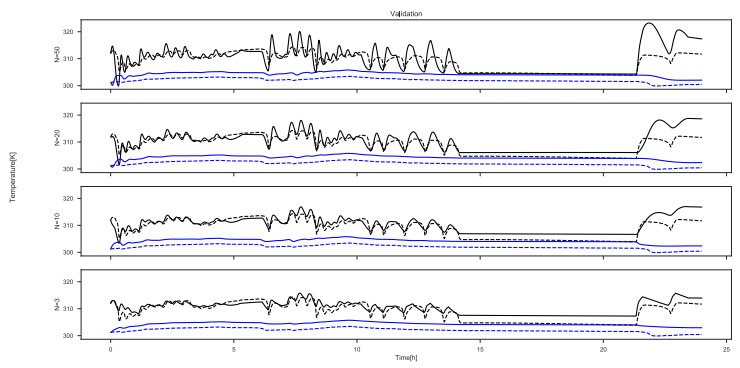

Figure 8. Validated model fit for the distributed model. Sensor signals $\left(T_{1}^{\mathrm{s}}, T_{2}^{\mathrm{s}}\right)$ : dashed lines. Simulated result: solid lines. Black color: $T_{1}^{\mathrm{s}}$, blue color: $T_{2}^{\mathrm{s}}$.

age in buildings. This paper discusses a well mixed tank model, and a distributed model which includes the effect of stratification. A buoyant conductivity term is included to handle buoyancy, as in (Xu et al., 2014); this model is hardly perfect, though. ${ }^{3}$ An alternative description would be that of natural convection as in (Vrettos, 2016).

Experimental data from a well instrumented new building is used to tune model parameters and validate the models. Initial results indicate that the well mixed model is too simple, in that model parameters depend considerably on the initial temperature distribution in the tank. The distributed model is discretized in $n$ slices (hyper parameter $^{4}$ ), where $n \in\{3,10,20,50\}$, and these are fitted to the data. The estimated parameters for the various values of $n$ are somewhat strange, in that between $n=10$ and $n=20$, parameters $k_{\mathrm{t}}$ (water conductivity) and $c_{\mathrm{b}}$ (buoyancy scaling parameter) switch values. However, remembering that coarse discretization in space gives an added mixing effect (adds to $k_{\mathrm{t}}$ ) while a finer discretization gives less such mixing, this may partially explain the variation in $k_{\mathrm{t}}$ estimates. Also, somewhat surprisingly, the root mean squared error (RMSE) increases with the number of slices for the calibrated model, which is contrary to what is expected. This could be due to numeric problems with solving and fitting larger models. Similarly, the RMSE values for the validated models also vary somewhat unexpectedly. Still, for $n \in\{3,10\}$ the model fit is decent.

In future work, the buoyancy model should be reconsidered, sensor signals should be checked/re-calibrated, with a revisit of how to handle data at different sample frequencies. Then, parameter estimation should be checked, possibly also introducing regularization in the model fitting.

\section{References}

Thomas Berthou, Pascal Stabat, Salvazet Raphael, and Dominique Marchio. Development and validation of a gray box model to predict thermal behavior of occupied office buildings. Energy and Buildings, 74:91-100, 2014. doi:10.1016/j.enbuild.2014.01.038.

\footnotetext{
${ }^{3}$ See, e.g., https://www.youtube.com/watch?v=wqtFeAvDOwk.

${ }^{4} \mathrm{~A}$ hyper parameter is a user selectable parameter.
}

Vinicius de Oliveira, Johannes Jäschke, and Sigurd Skogestad. Dynamic online optimization of a house heating system in a fluctuating energy price scenario. IFAC Proceedings Volumes, 46(32):463-468, December 2013. doi:10.3182/20131218-3IN-2045.00070.

Samuel F. Fux, Araz Ashouri, Michael J. Benz, and Lino Guzzella. Ekf based self-adaptive thermal model for a passive house. Energy and Buildings, 68:811-817, 2012. ISSN 0378-7788. doi:10.1016/j.enbuild.2012.06.016.

Casper Amandus Johansen. Evaluation of control systems with models for thermal energy in buildings. Master's thesis, University of South-Eastern Norway, P.O. Box 203, N-3901 Porsgrunn, Norway, May 2019. External partner: Autobolig AS.

Stephan Koch. Demand response methods for ancillary services and renewable energy integration in electric power systems. PhD thesis, ETH, Power Systems Laboratory, 2012.

Bernt Lie. Improved model for solar heating of buildings. In Lena Buffoni, Adrian Pop, and Bernhard Thiele, editors, Proceedings, The 56th Conference of Simulation and Modelling, pages 299-308, Linköping University, Sweden, October 2015. Scandinavian Simulation Society, Linköping University Electronic Press. doi:10.3384/ecp15119, pp. 299308. October 7-9 2015.

Bernt Lie, Carlos Pfeiffer, Nils-Olav Skeie, and Hans-Georg Beyer. Models for solar heating of buildings. In Alireza Rezania Kolai, Kim Sørensen, and Mads Pagh Nielsen, editors, Proceedings, 55th International Conference of Scandinavian Simulation Society, pages 28-38, Aalborg University, Denmark, October 2014. Scandinavian Simulation Society, Linköping University Electronic Press. doi:www.ep.liu.se/ecp/108/ecp14108.pdf. October 21-22 2014.

Luis Pérez-Lombard, José Ortiz, and Christine Pout. A review on buildings energy consumption information. Energy and Buildings, 40:394-398, 2008. doi:10.1016/j.enbuild.2007.03.007.

R. Viskanta, M. Behnia, and A. Karalis. Interferometric observations of the temperature structure in water cooled or heated from above. Advances in Water Resources, 1(2):57-69, 1977.

Evangelos Vrettos. Control of Residential and Commercial Loads for Power System Ancillary Services. PhD thesis, ETH, Power Systems Laboratory, 2016.

Zhijie Xu, Ruisheng Diao, Shuai Lu, Jianming Lian, and Yu Zhang. Modeling of electric water heaters for demand response: A baseline pde model. IEEE Transactions on Smart Grid, 5(5):2203-2210, September 2014. doi:10.1109/TSG.2014.2317149. 